\title{
Semitendinosus and gracilis free muscle-tendon graft for repair of massive rotator cuff tears: surgical technique
}

\author{
ANTONIO GIGANTE ${ }^{1}$, CARLO BOTTEGONI ${ }^{1}$, GIUSEPPE MILANO², MICHELE RICCIO³, \\ LUCA DEI GIUDICI ${ }^{1}$
}

1 Clinical Orthopaedics, Department of Clinical and Molecular Science DISCLIMO, Polytechnic University of Marche, Ancona, Italy

2 Department of Orthopaedics, Catholic University, "A. Gemelli" University Hospital, Rome, Italy

3 Department of Reconstructive Plastic Surgery - Hand Surgery, AOU "Ospedali Riuniti”, Ancona, Italy

\begin{abstract}
Massive rotator cuff tears are difficult to treat surgically due to retraction, degeneration and fraying of the ends of torn tendons, severe fatty infiltration and atrophy of the respective muscles.

Procedures developed to close the gap between the rotator cuff and the greater tuberosity of the humerus, such as soft tissue release may be inadequate for large tears. Human or porcine dermal allografts still have uncertain benefits, and tendon transfers seem to be associated with poor outcomes, donor site morbidity and altered mechanics. Reverse total shoulder arthroplasty has limited durability and is not indicated in young patients with high functional demands. We developed a new technique for repairing massive rotator cuff tears by semitendinosus and gracilis myotendinous grafting. This novel therapeutic option allows massive rotator cuff tears to be repaired using autologous material that is adequate and adaptable, making it possible to cover any width of defect. The technique is low-invasive and not technically demanding, with minimal donor site morbidity.
\end{abstract}

Keywords: rotator cuff tear, rotator cuff repair, rotator cuff augmentation, tendon transfer.

\footnotetext{
Corresponding Author:

Antonio Gigante, MD, PhD

Department of Clinical and Molecular Science

DISCLIMO, Università Politecnica delle Marche

Via Tronto 10/A, 60126 Ancona, Italy

E-mail: a.gigante@univpm.it
}

\section{Introduction}

Massive rotator cuff tears are difficult to treat surgically due to a series of problems that make repair by conventional methods inadequate, namely retraction, degeneration and fraying of torn tendon ends, severe fatty infiltration, and atrophy of the respective muscles. These tears are frequently irreparable due to the considerable width of the lesion, which often involves the supraspinatus and infraspinatus tendons; even if direct repairs are structurally successful, recurrence of the rupture after treatment is common.

Procedures developed to close the gap between the rotator cuff and greater tuberosity of the humerus, such as soft tissue release are inadequate for large tears $(>5 \mathrm{~cm})$. Autologous fascia lata and free biceps tendon grafts have been proposed but differences between surgical techniques, together with the lack of randomized clinical trials and long-term follow-up, preclude clear interpretation of the results (1). When there is only minimal or no fatty muscle infiltration present, but there is insufficient tendon for a repair, a human or porcine dermal allograft may augment the repair and enhance healing; however, the benefits of this approach remain uncertain due to related intolerance, inflammation and complications (2). Tendon transfers, such as latissimus dorsi flaps, seem to lead to donor site morbidity, altered mechanics after the transfer, and the need for specific and long rehabilitation, and they show poor outcomes (3). We developed a new surgical technique for repairing massive rotator cuff tears by means of semitendinosus (ST) and gracilis (G) myotendinous grafting as biological augmentation. The main elements contributing to the rationale for this choice are: i) the observation that the wide ST-G myo- 
tendinous junction appears appropriate for repairing a classical triangle-shaped defect (4); ii) the amount of muscle tissue usually present in the proximal portion of those tendons after their harvesting, regardless of the stripper used (4), and iii) the low postoperative donor site morbidity (5). Our choice was also supported by evidence of the use of this approach for the repair of other tendons, such as the patellar, tibialis anterior (6) and biceps brachii (5) tendons.

\section{Surgical technique}

The patient is placed in the beach chair position; the first step is a standard shoulder arthroscopy, performed in order to confirm the diagnosis of a massive lesion of the supraspinatus and infraspinatus, to test its reducibility, and to perform accurate release of adhesions between the ruptured tendons and other soft tissues. After trimming the edge of the torn tendons, two simple wires are inserted at the myotendinous junction to pull the muscles and fix the graft. The supraspinatus and infraspinatus are then each given two non-absorbable "U" knot sutures, to serve as a connection with the graft (Fig. 1).

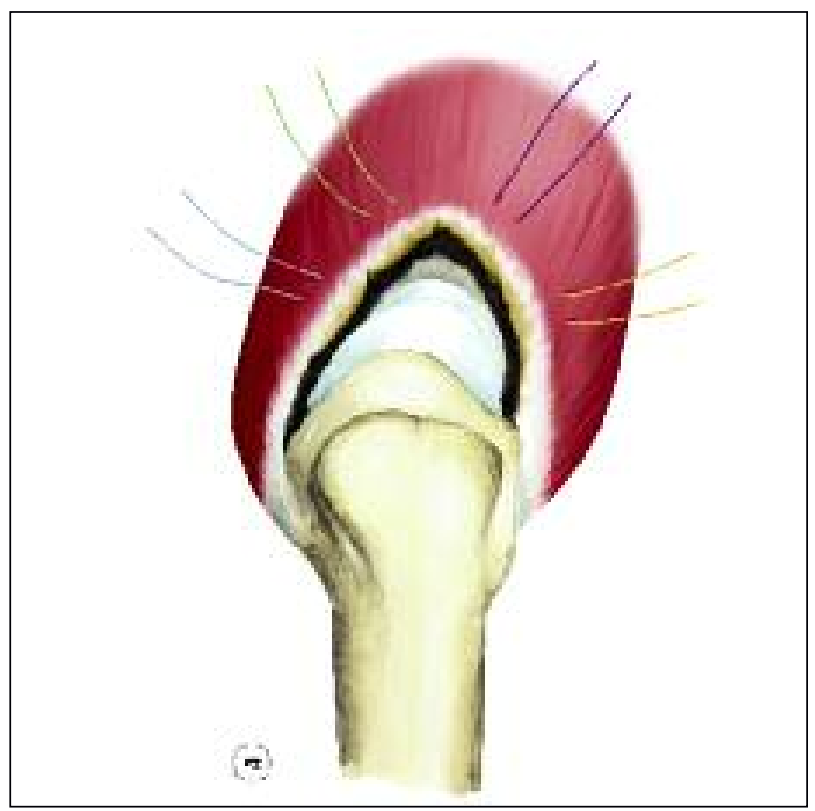

Fig. 1. Schematic drawing illustrating a massive rotator cuff tear. Muscles are given simple sutures that will be used to connect the "fan-like" portion of the graft to the rotator cuff.
The surgical incision, $5 \mathrm{~cm}$ long, is then made parallel to the deltoid muscle fibers, from $2-3 \mathrm{~cm}$ proximal to $1-2 \mathrm{~cm}$ distal to the anterolateral corner of the acromion). The humeral head is exposed and the tendon footprint is shaved and drilled until a regular and bleeding area with a mean size of $2 \times 1.5 \mathrm{~cm}^{2}$ is obtained. The ST-G tendons are then harvested in a standard fashion, the proximal portion of the tendons is smoothed on the workstation, maintaining as much muscle tissue as possible, reproducing a "fan like" proximal end, with a superior aspect of tendon tissue and an inferior aspect of muscle tissue. During these procedures, the muscle tissue should be hydrated in saline solution.

The free myotendinous grafts are finally joined together, along one of their edges with few simple knots of resorbable suture, in such a way that one aspect consists of muscular tissue and the other of tendon tissue (Fig. 2).

The "fan-like" portion is then sutured above the ruptured tendons with two simple knots of non-absorbable sutures just before the myotendinous junction of the cuff. We interpose autologous platelet rich plasma matrix between muscular aspect of the graft and the muscular aspect of the cuff, but the technique can also be performed without this biological stimulus. The free ends of the afore mentioned "U" knots are then passed into the graft. In order to test the strength of the sutures, the distal part of the ST-G tendons is gently pulled lengthwise. Proper tensioning is checked. A first metal anchor with two high-resistance sutures is placed in the prepared footprint to obtain primary stabilization of the graft, and a second anchor is then placed to achieve more stability and to obtain a footprint-wide reinsertion (Fig. 3). The graft is large enough to bridge the gap and cover the ends of the torn cuff tendons; the tubular parts of the graft can be used to fill minimal residual gaps if present (Fig. 4). The shoulder is taken through its full range of motion, making sure there is no restriction to terminal abduction, flexion and rotation. The incisions are closed with absorbable sutures on the fascia and the subcutaneous planes. The fascia overlying the pes anserinus is then closed with absorbable sutures. The skin is closed with non-absorbable sutures. A sling brace at $20^{\circ}$ $30^{\circ}$ of abduction should be adopted in the postoperative period for 30 days in total, starting from the moment the patient is discharged from the operating theater. 


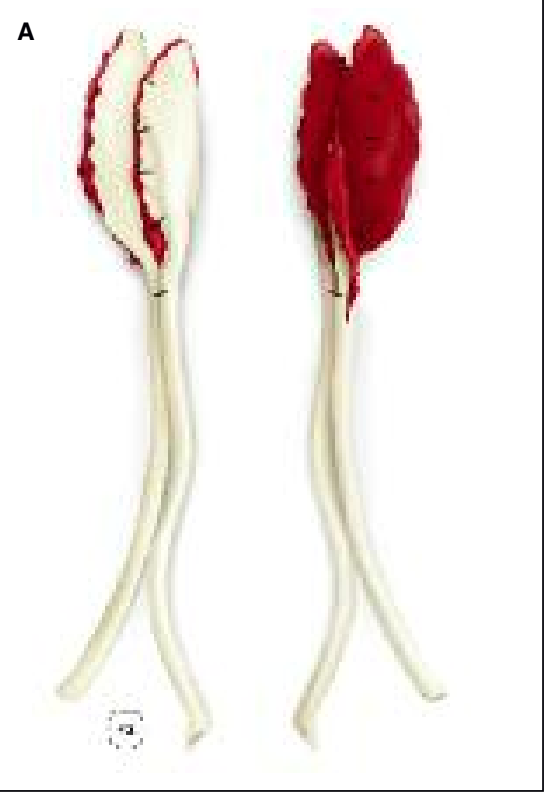

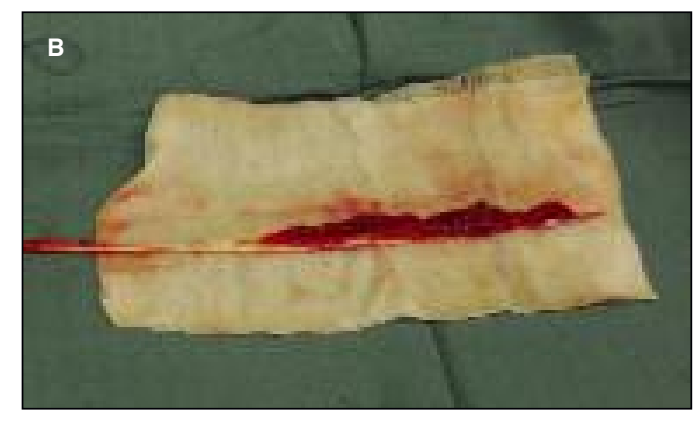

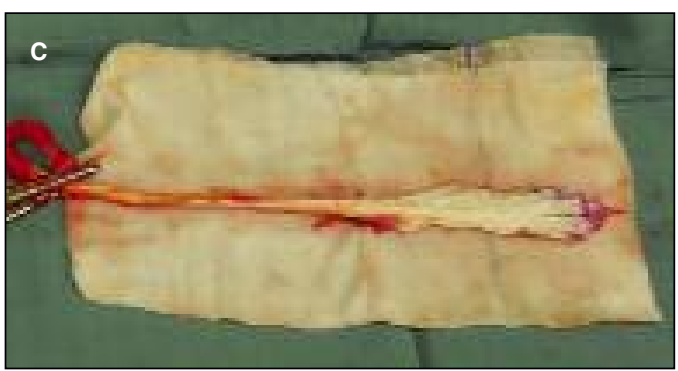

Fig. 2. A: Illustration of the prepared graft. The semitendinosus and gracilis tendons are used in an uncommon way compared to usual procedures. The myotendinous porres. The myotendinous por-
tions are only, splayed to
resemble a "fan", and joined resemble a "fan", and joined such a way that one aspect consists almost solely of muscle tissue and the other of of a stripped tendon under preparation. The inferior aspect, rich in muscular tissue, can be noted. C: Picture of a stripped tendon under preparation. The superior aspect, made up of tendon tissue, can be noted.

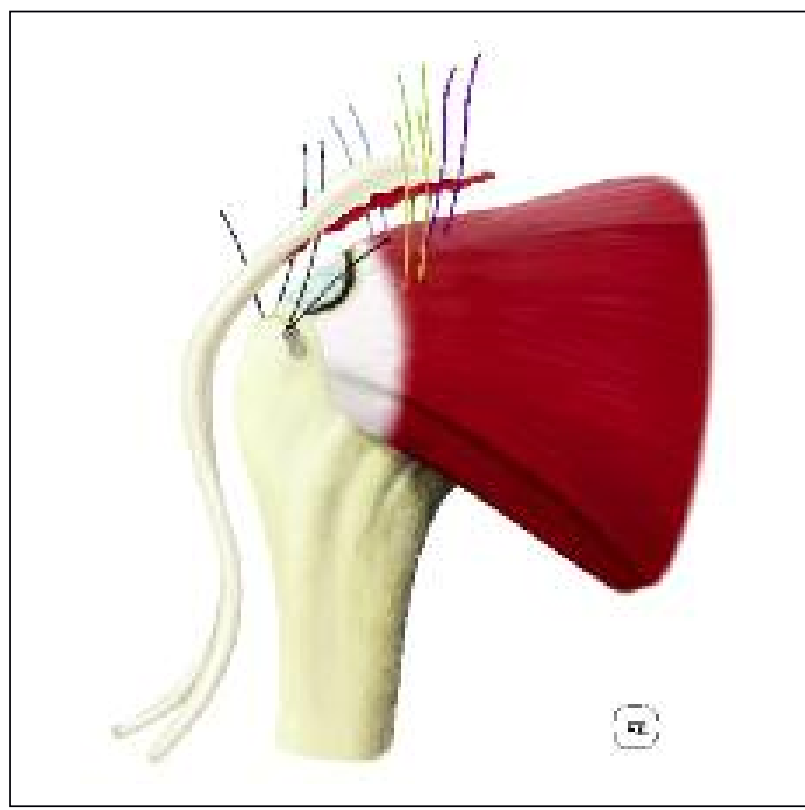

Fig. 3. Schematic representation of the augmentation from an anterior perspective, before tying the knots. Note the biggest innovation of this procedure: the muscular aspect of the prepared graft is positioned in a way that will bring it in contact with the muscular tissue of the rotator cuff, and will be attached to it by the four sutures applied in the first passage. The opposite end of the "fan" will serve, instead as traction tissue for the high-resistance sutures coming from the anchors and will be sutured to the rotator cuff tendon.

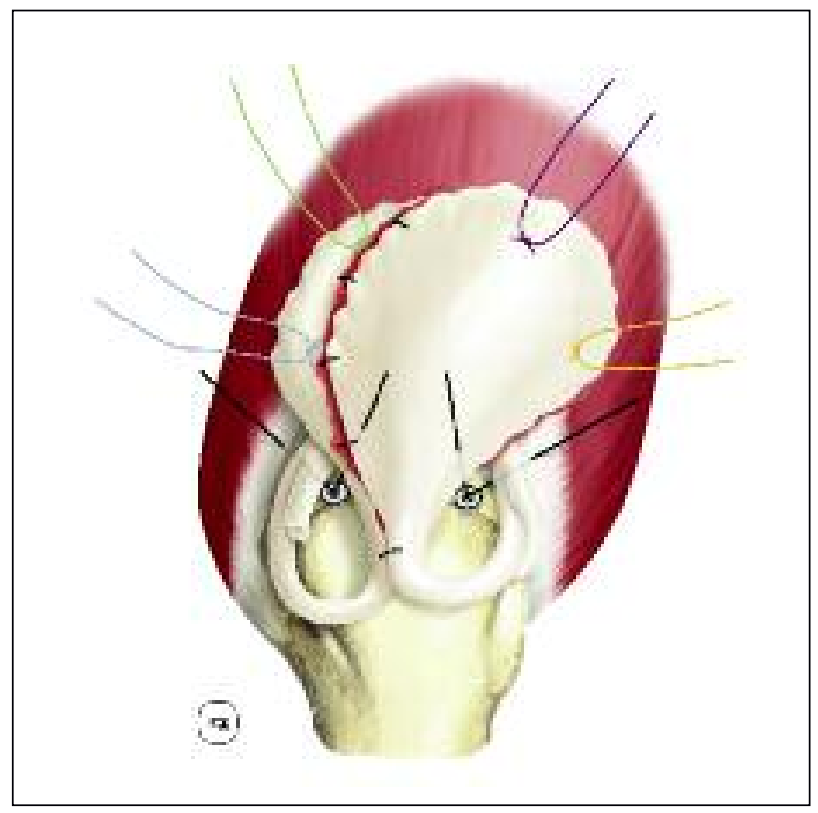

Fig 4. Final appearance of the proposed augmented repair. Graft free ends can be used to cover any lateral gaps that are left unfilled by an excessively narrow "fan" terminal portion. 